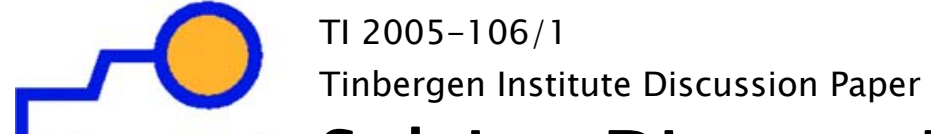 Solving Discrete Zero Point Problems with Vector Labeling
}

\author{
Gerard van der Laan' \\ Dolf Talman² \\ Zaifu Yang
}

${ }^{\prime}$ Department of Econometrics, Vrije Universiteit Amsterdam, and Tinbergen Institute, 2 Department of Econometrics \& Operations Research, and CentER, Tilburg University,

${ }^{3}$ Faculty of Business Administration, Yokohama National University, Japan. 


\section{Tinbergen Institute}

The Tinbergen Institute is the institute for economic research of the Erasmus Universiteit Rotterdam, Universiteit van Amsterdam, and Vrije Universiteit Amsterdam.

Tinbergen Institute Amsterdam

Roetersstraat 31

1018 WB Amsterdam

The Netherlands

Tel.: $\quad+31(0) 205513500$

Fax: $\quad+31(0) 205513555$

Tinbergen Institute Rotterdam

Burg. Oudlaan 50

3062 PA Rotterdam

The Netherlands

Tel.: $\quad+31(0) 104088900$

Fax: $\quad+31(0) 104089031$

Please send questions and/or remarks of nonscientific nature to driessen@tinbergen.nl.

Most TI discussion papers can be downloaded at http://www.tinbergen.nl. 


\title{
Solving Discrete Zero Point Problems with Vector Labeling ${ }^{1}$
}

\author{
Gerard van der Laan, ${ }^{2}$ Dolf Talman, ${ }^{3}$ Zaifu Yang ${ }^{4}$
}

November 24, 2005

${ }^{1}$ This research was carried out while Gerard van der Laan and Zaifu Yang were visiting the CentER for Economic Research, Tilburg University. The visit of Zaifu Yang has been made possible by financial support of CentER and the Netherlands Organization for Scientific Research (NWO).

${ }^{2}$ G. van der Laan, Department of Econometrics and Tinbergen Institute, Vrije Universiteit, De Boelelaan 1105, 1081 HV Amsterdam, The Netherlands, glaan@feweb.vu.nl

${ }^{3}$ A.J.J. Talman, Department of Econometrics \& Operations Research and CentER, Tilburg University, P.O. Box 90153, 5000 LE Tilburg, The Netherlands, talman@uvt.nl

${ }^{4}$ Z. Yang, Faculty of Business Administration, Yokohama National University, Yokohama 2408501, Japan, yang@ynu.ac.jp 


\begin{abstract}
In this paper we present two general results on the existence of a discrete zero point of a function from the $n$-dimensional integer lattice $\mathbb{Z}^{n}$ to the $n$-dimensional Euclidean space $\mathbb{R}^{n}$. Under two different boundary conditions, we give a constructive proof using a combinatorial argument based on a simplicial algorithm with vector labeling and lexicographic linear programming pivot steps. We also adapt the algorithm to prove the existence of a solution to the discrete complementarity problem.
\end{abstract}

Keywords: Integer lattice, zero point, vector labeling rule, simplicial algorithm, BorsukUlam, discrete complementarity.

AMS subject classification: 47H10, 54H25, 55M20, 90C26, 90C33, 91B50.

JEL classification: C61, C62, C68, C72, C58. 


\section{Introduction}

We consider the problem of finding a point $x^{*} \in \mathbb{Z}^{n}$ such that

$$
f\left(x^{*}\right)=0^{n}
$$

where $0^{n}$ is the $n$-vector of zeroes, $Z^{n}$ is the integer lattice of the $n$-dimensional Euclidean space $\mathbb{R}^{n}$, and $f$ is a function from $\mathbb{Z}^{n}$ to $\mathbb{R}^{n}$. Such an integral point $x^{*}$ is called a discrete zero point of $f$. Recently, the existence problem of an integral solution has been investigated in several papers. These papers were all inspired by the discrete fixed point statement given in Iimura [10]. The existence theorems in Iimura, Murota and Tamura [11] and Danilov and Koshevoy [4] concern functions that exhibit the so-called direction-preserving property, which can be seen as the counterpart of the continuity property for functions defined on the Euclidean space $\mathbb{R}^{n}$. The existence results in Yang [33] and [34] hold for the class of so-called locally gross direction-preserving mappings, which is substantially more general and richer than the class of limura's direction-preserving mappings and which contains the results in [4] and [11] as special cases. Besides establishing these more general existence results, Yang also initiated in [33] the study of discrete nonlinear complementarity problems and provided several general theorems for the existence of solutions for this class of problems. All this literature, however, is not concerned with the problem of finding an integral solution. In fact, all these existence proofs are nonconstructive.

To provide constructive proofs based on a combinatorial argument we apply the technique of the so-called simplicial algorithms originally designed to find approximate zero or fixed points of continuous functions or upper semi-continuous mappings. The first of such algorithm was developed by Scarf [26] and subsequent algorithms proposed by Eaves [6], Eaves and Saigal [7], Merrill [22], van der Laan and Talman [16] among others, substantially improved Scarf's original one in term of efficieny and applicability. For comprehensive treatments on such algorithms we refer to Allgower and Georg [1], Todd [27] and Yang [32]. In van der Laan, Talman and Yang [19], [20], the so-called $2 n$ ray integer labeling simplicial algorithm independently introduced by van der Laan and Talman [17] and Reiser [25], has been modified to find an integral solution of functions satisfying the direction-preserving property and to find an integral solution for directionpreserving discrete nonlinear complementarity problems, respectively.

The aim of this paper is to provide a combinatorial algorithm for finding an integral solution of functions satisfying the more general locally gross direction-preserving property. This algorithm is again a modification of the $2 n$-ray simplicial algorithm, introduced in [17] and [25]. However, in this case we cannot rely on integer labeling anymore, instead we have to apply the more subtle concept of vector labeling. The modified algorithm makes use of a triangulation of $\mathbb{R}^{n}$, being a family of integral simplices, constructed in such a way that 
the set of vertices of the simplices of the triangulation is equal to $\mathbb{Z}^{n}$ and the mesh size of each simplex in the triangulation is equal to one according to the maximum norm. Starting with some integral point in $\mathbb{Z}^{n}$, the algorithm leaves the starting point along one out of $2 n$ directions and then generates a sequence of adjacent simplices of varying dimension by making lexicographic linear programming pivot steps in a system of linear equations. For the existence of a zero point of a function on the $n$-dimensional Euclidean space $\mathbb{R}^{n}$ some kind of boundary condition is needed in order to restrict the feasible domain of the function to a convex and compact set in $\mathbb{R}^{n}$. Similarly, we also need boundary conditions for the discrete case. We provide two different boundary conditions, both guaranteeing that the algorithm terminates within a finite number of steps with a simplex having one of its vertices as an integral solution to the zero point problem. In addition, we adapt the algorithm to prove the existence of a solution to the discrete nonlinear complementarity problem. Whereas all the previous works on the computation of fixed or zero points of a continuous function find only an approximate solution, when applied to the current discrete case, the $2 n$-ray algorithm finds an exact solution.

This paper is organized as follows. In Section 2 we introduce the concept of triangulation and locally gross direction preservation and we describe the algorithm. In Sections 3 and 4 we state two boundary conditions guaranteeing an integral solution of the discrete zero-point problem and provide constructive proofs based on the combinatorial argument that the algorithm will find a solution within a finite number of steps. In Section 5 we apply the algorithm to find an integral solution to the discrete complementarity problem.

\section{Triangulation and algorithm}

For a given positive integer $n$, let $N$ denote the set $\{1,2, \ldots, n\}$. For $i \in N, e(i)$ denotes the $i$ th unit vector of $\mathbb{R}^{n}$. Given a set $D \subset \mathbb{R}^{n}, \operatorname{Co}(D)$ and $\operatorname{Bd}(D)$ denote the convex hull of $D$ and the (relative) boundary of $D$, respectively. For any $x$ and $y$ in $\mathbb{R}^{n}$, we say $y$ is lexicographically greater than $x$, and denote it by $y \succeq x$, if the first nonzero component of $y-x$ is positive.

Two integral points $x$ and $y$ in $\mathbb{Z}^{n}$ are said to be cell-connected if $\max _{h \in N}\left|x_{h}-y_{h}\right| \leq$ 1, i.e., their distance is less than or equal to one according to the maximum norm. In other words, two integral points $x$ and $y$ are cell-connected if and only if there exists $q \in \mathbb{Z}^{n}$ such that both $x$ and $y$ belong to the hyper cube $[0,1]^{n}+\{q\}$.

For an integer $t, 0 \leq t \leq n$, the $t$-dimensional convex hull of $t+1$ affinely independent points $x^{1}, \ldots, x^{t+1}$ in $\mathbb{R}^{n}$ is called a $t$-simplex or simply a simplex and will be denoted by $<x^{1}, \ldots, x^{t+1}>$. The extreme points $x^{1}, \ldots, x^{t+1}$ of a $t$-simplex $\sigma=<x^{1}, \ldots, x^{t+1}>$ are called the vertices of $\sigma$. The convex hull of any subset of $k+1$ vertices of a $t$-simplex $\sigma$, 
$0 \leq k \leq t$, is called a face or $k$-face of $\sigma$. A $k$-face of a $t$-simplex $\sigma$ is called a facet of $\sigma$ if $k=t-1$, i.e., if the number of vertices is just one less than the number of vertices of the simplex. A simplex is said to be integral if all of its vertices are integral vectors and are cell-connected. Any two vertices $x$ and $y$ of an integral simplex are said to be simplicially connected.

Given an $m$-dimensional convex set $D$, a collection $\mathcal{T}$ of $m$-dimensional simplices is a triangulation or simplicial subdivision of the set $D$, if (i) $D$ is the union of all simplices in $\mathcal{T}$, (ii) the intersection of any two simplices of $\mathcal{T}$ is either empty or a common face of both, and (iii) any neighborhood of any point in $D$ only meets a finite number of simplices of $\mathcal{T}$. A triangulation is called integral if all its simplices are integral simplices.

Now we introduce the class of locally gross direction preserving functions on which the existence theorems of this paper are based. Locally gross direction preservation replaces continuity of a function defined on $\mathbb{R}^{n}$. Let $a \cdot b$ denote the inner product of two vectors $a$ and $b$ in $\mathbb{R}^{n}$.

\section{Definition 2.1}

(i) A function $f: \mathbb{Z}^{n} \rightarrow \mathbb{R}^{n}$ is locally gross direction preserving if for any cell-connected points $x$ and $y$ in $\mathbb{Z}^{n}$ it holds that

$$
f(x) \cdot f(y) \geq 0
$$

(ii) A function $f: \mathbb{Z}^{n} \rightarrow \mathbb{R}^{n}$ is simplicially local gross direction preserving with respect to some given integral triangulation $\mathcal{T}$ of $\mathbb{R}^{n}$, if for any vertices $x$ and $y$ of every simplex of $\mathcal{T}$ it holds that

$$
f(x) \cdot f(y) \geq 0
$$

The locally gross preserving property was originally introduced in Yang [34] and prevents the function from changing too drastically in direction within one cell. The simplicially local gross preserving condition is weaker and only requires that the function does not change too drastically in direction within any integral simplex of the given triangulation. Since any two vertices of a simplex of an integral triangulation are cell-connected we have the property that every locally gross direction preserving function is also simplicially local gross direction preserving with respect to any integral triangulation.

To compute a discrete zero point of a (simplicially) local gross direction preserving function, we adapt the $2 n$-ray vector labeling algorithm introduced in van der Laan and Talman [17], see also Reiser [25] for integer labeling, to the current discrete setting. This algorithm was originally proposed to obtain an approximate fixed point of a continuous function. 
Let $f$ be a function being simplicially local gross direction preserving with respect to some given integral triangulation $\mathcal{T}$. Recall that if $f$ is locally gross direction preserving, then we can take any integral triangulation $\mathcal{T}$. Let $v$ be an arbitrarily chosen integral vector in $\mathrm{Z}^{n}$. The point $v$ will be the starting point of the algorithm. For a nonzero sign vector $s \in\{-1,0,+1\}^{n}$, the subset $A(s)$ of $\mathbb{R}^{n}$ is defined by

$$
A(s)=\left\{x \in \mathbb{R}^{n} \mid x=v+\sum_{h \in N} \alpha_{h} s_{h} e(h), \alpha_{h} \geq 0, h \in N\right\} .
$$

Clearly, the set $A(s)$ is a $t$-dimensional subset of $\mathbb{R}^{n}$, where $t$ is the number of nonzero components of the sign vector $s$, i.e., $t=\left|\left\{i \mid s_{i} \neq 0\right\}\right|$. Since $\mathcal{T}$ is an integral triangulation of $\mathbb{R}^{n}$, it triangulates every set $A(s)$ into $t$-dimensional integral simplices. For some $s$ with $t$ nonzero components, denote $\left\{h_{1}, \cdots, h_{n-t}\right\}=\left\{h \mid s_{h}=0\right\}$ and let $\sigma=<x^{1}, \cdots, x^{t+1}>$ be a $t$-simplex of the triangulation in $A(s)$. Following Todd [29], who improved the original system of equations used by van der Laan and Talman [17], we say that $\sigma$ is almost $s$ complete if there is an $(n+2) \times(n+1)$ matrix $W$ satisfying

$$
\left[\begin{array}{ccccccc}
1 & \cdots & 1 & 0 & \cdots & 0 & 1 \\
f\left(x^{1}\right) & \cdots & f\left(x^{t+1}\right) & e\left(h_{1}\right) & \cdots & e\left(h_{n-t}\right) & -s
\end{array}\right] W=I
$$

and having rows $w^{1}, \cdots, w^{n+2}$ such that $w^{h} \succeq 0$ for $1 \leq h \leq t+1, w^{n+2} \succeq w^{i}$ and $w^{n+2} \succeq-w^{i}$ for $t+1<i \leq n+1$, and $w^{n+2} \succeq 0$. Here $I$ denotes the identity matrix of rank $n+1$. If $w_{1}^{n+2}=0$, then we say that the simplex $\sigma$ is complete. Further, let $\tau$ be a facet of $\sigma$, and, without loss of generality, index the vertices of $\sigma$ such that $\tau=\left\langle x^{1}, \cdots, x^{t}\right\rangle$. We say that $\tau$ is $s$-complete if there is an $(n+1) \times(n+1)$ matrix $W$ satisfying

$$
\left[\begin{array}{ccccccc}
1 & \cdots & 1 & 0 & \cdots & 0 & 1 \\
f\left(x^{1}\right) & \cdots & f\left(x^{t}\right) & e\left(h_{1}\right) & \cdots & e\left(h_{n-t}\right) & -s
\end{array}\right] W=I
$$

and having rows $w^{1}, \cdots, w^{n+1}$ such that $w^{h} \succeq 0$ for $1 \leq h \leq t, w^{n+1} \succeq w^{i}$ and $w^{n+1} \succeq-w^{i}$ for $t+1 \leq i \leq n$, and $w^{n+1} \succeq 0$. If $w_{1}^{n+1}=0$, then we say that $\tau$ is complete.

The lemma below says that the 0-dimensional simplex $v$ is an $s$-complete facet for a uniquely determined sign vector containing one nonzero element. Let $\alpha=\max _{h}\left|f_{h}(v)\right|$. Now we define the sign vector $s^{0}$ as follows. If $f_{h}(v)=-\alpha$ for some $h$, then we take $s_{k}^{0}=-1$ where $k$ is the smallest index $h$ such that $f_{h}(v)=-\alpha$, and $s_{j}^{0}=0$ for $j \neq k$. For example, if $f(v)=0^{n}$, then $s^{0}=(-1,0, \cdots, 0)$. In case $f_{h}(v)>-\alpha$ for all $h$, then we take $s_{k}^{0}=1$ where $k$ is the largest index $h$ such that $f_{h}(v)=\alpha$, and $s_{j}^{0}=0$ for $j \neq k$. Let $\sigma^{0}$ be the unique 1-dimensional simplex in $A\left(s^{0}\right)$ containing $\langle v\rangle$ as a facet.

Lemma 2.2 The simplex $\langle v\rangle$ is an $s^{0}$-complete facet of $\sigma^{0}$. 
Proof. Consider the system

$$
\left[\begin{array}{cccccccc}
1 & 0 & \cdots & 0 & 0 & \cdots & 0 & 1 \\
f(v) & e(1) & \cdots & e(k-1) & e(k+1) & \cdots & e(n) & -s_{k}^{0} e(k)
\end{array}\right] V=I .
$$

By the structure of the first left-hand matrix it follows that its inverse $V$ exists and that its rows $v^{1}, \ldots, v^{n+1}$ are given by

$$
\begin{aligned}
& v^{1}=(1,0, \cdots, 0), \\
& v^{h}=\left(f_{k-1}(v), 0, \cdots, 0,-1,0, \cdots, 0\right), \quad h=2, \ldots, k,
\end{aligned}
$$

with -1 being the $h$ th component,

$$
v^{h}=\left(f_{k-1}(v), 0, \cdots, 0,-1,0, \cdots, 0\right), \quad h=k+1, \ldots, n,
$$

with -1 being the $(h+1)$ th component and

$$
v^{n+1}=\left(s_{k}^{0} f_{k}(v), 0, \cdots, 0,-s_{k}^{0}, 0, \cdots, 0\right)
$$

with $-s_{k}^{0}$ being the $(k+1)$ th component. Clearly, $v^{1}$ is lexicographically positive. Moreover, $v^{n+1}$ is lexicographically positive, because we have either $s_{k}^{0} f_{k}(v)>0$ or $s_{k}^{0} f_{k}(v)=0$ and $-s_{k}^{0}>0$. For $j=2, \cdots, k$, we have $v^{n+1} \succeq v^{j}$, because $s_{k}^{0} f_{k}(v) \geq f_{j-1}(v)$, and we also have $v^{n+1} \succeq-v^{j}$, because $f_{j-1}(v)>-s_{k}^{0} f_{k}(v)$. For $j=k+1, \cdots, n$, we have $v^{n+1} \succeq v^{j}$, because either $s_{k}^{0} f_{k}(v)>f_{j}(v)$ or $s_{k}^{0} f_{k}(v)=f_{j}(v)$ and $s_{k}^{0}=-1$, and we also have $v^{n+1} \succeq-v^{j}$, because either $s_{k}^{0} f_{k}(v)>-f_{j}(v)$ or $s_{k}^{0} f_{k}(v)=-f_{j}(v)$ and $s_{k}^{0}=-1$. Hence, $V$ satisfies all the requirements of the matrix $W$ in system (2.2) and thus $\langle v\rangle$ is an $s^{0}$-complete facet of $\sigma^{0}$.

We are now able to describe the algorithm for finding an integral solution to the function $f$. When for some nonzero sign vector $s$ a $t$-simplex $\sigma=<x^{1}, \cdots, x^{t+1}>$ in $A(s)$ is almost $s$-complete, the system (2.1) has two "basic solutions". At each of these solutions exactly one condition on the rows of the solution $W$ is binding. If $w^{h} \succeq 0$ is binding for some $h, 1 \leq h \leq t+1$, then the facet $\tau$ of $\sigma$ opposite the vertex $x^{h}$ is $s$-complete, then, if not complete, $\tau$ is either (i) the 0-dimensional simplex $\langle v\rangle$ or (ii) a facet of precisely one other almost $s$-complete $t$-simplex $\sigma^{\prime}$ of the triangulation in $A(s)$ or (iii) lies on the boundary of $A(s)$ and is an almost $s^{\prime}$-complete $(t-1)$-simplex in $A\left(s^{\prime}\right)$ for some unique nonzero sign vector $s^{\prime}$ with $t-1$ nonzero elements differing from $s$ in only one element. If $w^{n+2} \succeq 0$ is binding, then $w_{1}^{n+2}=0$ and $\sigma$ must be complete. Further, if $w^{n+2} \succeq w^{i}$ $\left(w^{n+2} \succeq-w^{i}\right)$ for some $t+1<i \leq n+1$ and, if not complete, $\sigma$ is an $s^{\prime}$-complete facet of precisely one almost $s^{\prime}$-complete $(t+1)$-simplex in $A\left(s^{\prime}\right)$ for some nonzero sign vector $s^{\prime}$ differing from $s$ in only the $i$ th element, namely $s_{i}^{\prime}=+1(-1)$. 
Since $\langle v\rangle$ is $s^{0}$-complete, $\sigma^{0}$ is an almost $s^{0}$-complete 1-dimensional simplex in $A\left(s^{0}\right)$. Starting with $\sigma^{0}$ the $2 n$-ray algorithm generates a sequence of adjacent almost $s$-complete simplices in $A(s)$ with $s$-complete common facets for varying sign vectors $s$. Moving from one $s$-complete facet to the next $s^{\prime}$-complete facet corresponds to making a lexicographic linear programming pivot step from one of the two basic solutions of system (2.1) to the other one. The algorithm stops as soon as it finds a complete simplex. We will show that in that case one of its vertices is a discrete zero point of the function $f$.

Lemma 2.3 Suppose that $f$ is simplicially local gross direction preserving with respect to $\mathcal{T}$. Then any complete simplex contains a discrete zero point of the function $f$.

Proof. Let $x^{1}, \cdots, x^{k+1}$ be the vertices of a complete simplex $\sigma$ in $A(s)$ and let $t$ be the number of nonzeros in $s$. Notice that $k=t-1$ or $k=t$ depending on whether $\sigma$ is a $t$ simplex in $A(s)$ or a facet of a $t$-simplex in $A(s)$. From the system (2.1) it follows that there exists $\lambda_{1} \geq 0, \cdots, \lambda_{k+1} \geq 0$ with sum equal to one such that $\sum_{j=1}^{k+1} \lambda_{j} f\left(x^{j}\right)=0^{n}$. Let $j^{*}$ be such that $\lambda_{j^{*}}>0$. Then by premultiplying $f\left(x^{j^{*}}\right)$ on both sides of $\sum_{j=1}^{k+1} \lambda_{j} f\left(x^{j}\right)=0^{n}$, we obtain

$$
\lambda_{1} f\left(x^{1}\right) \cdot f\left(x^{j^{*}}\right)+\cdots+\lambda_{j^{*}} f\left(x^{j^{*}}\right) \cdot f\left(x^{j^{*}}\right)+\cdots+\lambda_{k+1} f\left(x^{k+1}\right) \cdot f\left(x^{j^{*}}\right)=0 .
$$

Since $f$ is simplicially local gross direction preserving, it is easy to see that every term in the above expression is nonnegative. Therefore every term is equal to zero. In particular, $f\left(x^{j^{*}}\right)=0^{n}$, and so $x^{j^{*}}$ is a discrete zero point of the function $f$.

In the next two sections we propose two different boundary conditions guaranteeing that the algorithm terminates within a finite number of steps.

\section{Convergence Condition I}

For $x \in \mathbb{Z}^{n}$, let $N(x)$ denote the set of integer points being cell-connected to $x$. Our first condition under which a discrete zero point will be found by the algorithm is given in the following assumption.

Assumption 3.1 Given a function $f: \mathbb{Z}^{n} \rightarrow \mathbb{R}^{n}$, there exist vectors $m, M \in \mathbb{Z}^{n}$, with $m_{h}<M_{h}-1$ for every $h \in N$, such that for every integral vector $x$ on the boundary of the set $C^{n}=\left\{z \in \mathbb{R}^{n} \mid m \leq z \leq M\right\}$ the following conditions hold:

(i) If $x_{i}=m_{i}$ then $f_{i}(y) \geq 0$ for all $y \in N(x) \cap C^{n}$ satisfying $y_{i}=m_{i}$ or there exists $j \in N$ such that $f_{j}(y)<f_{i}(y)$ for all $y \in N(x) \cap C^{n}$ satisfying $y_{i}=m_{i}$.

(ii) If $x_{i}=M_{i}$ then $f_{i}(y) \leq 0$ for all $y \in N(x) \cap C^{n}$ satisfying $y_{i}=M_{i}$ or there exists $j \in N$ such that $f_{j}(y)>f_{i}(y)$ for all $y \in N(x) \cap C^{n}$ satisfying $y_{i}=M_{i}$. 
The condition means that there exist lower and upper bounds, such that when $x$ is an integral vector on the $i$ th lower (upper) bound, then either $f_{i}(y)$ is nonnegative (nonpositive) for any integral vector $y$ on the same lower (upper) bound being cell-connected to $x$ or, for some $j \neq i, f_{i}(y)$ is larger (smaller) than $f_{j}(y)$ for any integral vector $y$ on the same lower (upper) bound being cell-connected to $x$. We show that under this condition any simplicially local gross direction preserving function has a discrete zero point within the bounded set induced by the lower and upper bounds.

Theorem 3.2 Let $f: \mathbb{Z}^{n} \rightarrow \mathbb{R}^{n}$ be a simplicially local gross direction preserving function with respect to some integral triangulation $\mathcal{T}$. When $f$ satisfies Assumption 3.1 , then $f$ has a discrete zero point in the set $C^{n}$.

Proof. Take any integral vector in the interior of the set $C^{n}$ as the starting point $v$ of the algorithm. By definition of integral triangulation, $\mathcal{T}$ triangulates the set $C^{n}$ and also the set $A(s) \cap C^{n}$ for any sign vector $s$ into integral simplices.

For some nonzero sign vector $s$, let $\tau$ be an $s$-complete facet in $A(s)$ with vertices $x^{1}, \cdots, x^{t}$, where $t$ is the number of nonzeros in $s$. We first show that $\tau$ is complete if it is on the boundary of $C^{n}$. From system (2.1) it follows that there exist $\lambda_{1} \geq 0, \cdots, \lambda_{t} \geq 0$ with sum equal to one, $\beta \geq 0$, and $-\beta \leq \mu_{i} \leq \beta$ for $s_{i}=0$, such that $\bar{f}_{i}(z)=\beta$ if $s_{i}=1, \bar{f}_{i}(z)=-\beta$ if $s_{i}=-1$, and $\bar{f}_{i}(z)=\mu_{i}$ if $s_{i}=0$, where $z=\sum_{i=1}^{t} \lambda_{i} x^{i}$ and $\bar{f}(z)=\sum_{i=1}^{t} \lambda_{i} f\left(x^{i}\right)$, i.e., $\bar{f}$ is the piecewise linear extension of $f$ with respect to $\mathcal{T}$. Since $\tau$ lies on the boundary of $C^{n}$, there exists an index $h$ such that either $x_{h}^{j}=m_{h}$ for all $j$ or $x_{h}^{j}=M_{h}$ for all $j$. In case $x_{h}^{j}=m_{h}$ for all $j$, we have $s_{h}=-1$ and therefore $\bar{f}_{h}(z)=-\beta$. Furthermore, by Assumption 3.1, we have (i) $f_{h}\left(x^{j}\right) \geq 0$ for all $j$ or (ii) there exists $k$ such that $f_{k}\left(x^{j}\right)<f_{h}\left(x^{j}\right)$ for all $j$. In case (ii) we have $f_{k}\left(x^{j}\right)<f_{h}\left(x^{j}\right)$ for all $j$. Hence $\bar{f}_{k}(z)<\bar{f}_{h}(z)$. On the other hand, $\bar{f}_{k}(z) \geq-\beta=\bar{f}_{h}(z)$, yielding a contradiction, i.e. this case cannot occur. In case (i) we obtain $\bar{f}_{h}(z) \geq 0$. On the other hand $\bar{f}_{h}(z)=-\beta \leq 0$. Therefore $\bar{f}_{h}(z)=0$ and also $\beta=0$. Since $w_{1}^{n+1}=\beta$ we obtain that $\tau$ is complete. Similarly, we can show that the same results hold for the case of $x_{h}^{j}=M_{h}$ for all $j$.

Now, consider the algorithm as described at the end of the previous section. Due to the lexicographic pivoting rule, the algorithm will never visit any simplex more than once. So, because the number of simplices in $C^{n}$ is finite, the algorithm finds within a finite number of steps either a complete simplex $\sigma$ or, for some $s$, it reaches an $s$-complete facet $\tau$ on the boundary of $C^{n}$. As shown above, also such a facet $\tau$ is complete. Hence, the algorithm finds a complete simplex in $C^{n}$ within a finite number of steps. Since $f$ is simplicially local gross direction preserving, Lemma 2.3 guarantees that at least one of the vertices of this complete simplex is a discrete zero point of the function $f$. 
Above we have provided a constructive proof of Theorem 3.2 based on the combinatorial argument that the algorithm ends within a finite number of steps with a simplex having one of its vertices as integral solution to $f$. Observe that the theorem implies the following corollary.

Corollary 3.3 Let $f: \mathbb{Z}^{n} \rightarrow \mathbb{R}^{n}$ be a simplicially local gross direction preserving function. Suppose that there exist vectors $m, M \in \mathbb{Z}^{n}$, with $m_{h}<M_{h}-1$ for every $h \in N$, such that for every integral vector $x$ on the boundary of the set $C^{n}=\left\{z \in \mathbb{R}^{n} \mid m \leq z \leq M\right\}$, $x_{i}=m_{i}$ implies $f_{i}(x) \geq 0$ and $x_{i}=M_{i}$ implies $f_{i}(x) \leq 0$. Then $f$ has a discrete zero point in $C^{n}$.

\section{Convergence Condition II}

Now we present a second condition under which a discrete zero point is shown to exist.

Assumption 4.1 Given a function $f: \mathbb{Z}^{n} \rightarrow \mathbb{R}^{n}$, there exists a vector $u \in \mathbb{Z}^{n}$ with $u_{h} \geq 1$ for all $h \in N$, such that $f(x) \cdot f(-y) \leq 0$ for any cell-connected integral points $x$ and $y$ lying on a same proper face of the set $U^{n}=\left\{z \in \mathbb{R}^{n} \mid-u \leq z \leq u\right\}$.

This boundary condition is very natural and can be viewed as a discrete analogue of a weak version of the Borsuk-Ulam antipodal condition for a continuous function saying that $f(x) \cdot f(-x) \leq 0$ when $x$ is on the boundary of $U^{n}$. It has been shown that under the latter condition a continuous function has a zero point; see for instance van der Laan [15] and Yang [32]. Todd and Wright [30] used a modification of the $2 n$-ray algorithm to give a constructive proof of the Borsuk-Ulam theorem and Freund and Todd [9] used the modified algorithm to give a constructive proof for a combinatorial lemma due to Tucker [31]. The following theorem was originally given in Yang [34].

Theorem 4.2 Let $f: \mathbb{Z}^{n} \rightarrow \mathbb{R}^{n}$ be a locally gross direction preserving function satisfying Assumption 4.1. Then $f$ has a discrete zero point in $U^{n}$.

In contrast to the nonconstructive proof in [34], below we give a constructive proof based on the combinatorial argument that the modified $2 n$-ray algorithm finds an integral solution within a finite number of steps. The proof is based on a lemma on the extension $V^{n}$ of the set $U^{n}$ given by

$$
V^{n}=\left\{x \in \mathbb{R}^{n} \mid-\left(u_{i}+1\right) \leq x_{i} \leq u_{i}+1, \quad \forall i \in N\right\} .
$$

Let the projection function $p: V^{n} \rightarrow U^{n}$ be defined by

$$
p_{h}(x)=\max \left\{-u_{h}, \min \left\{u_{h}, x_{h}\right\}\right\}, \text { for all } h \in N \text {. }
$$


Clearly, $p(x)=x$ if $x \in U^{n}$. Moreover, $p(x) \in U^{n} \cap \mathbb{Z}^{n}$ if $x \in V^{n} \cap \mathbb{Z}^{n}$. We now define the function $g: V^{n} \cap \mathbb{Z}^{n} \rightarrow \mathbb{R}^{n}$ by setting $g(x)=f(x)$ for $x \in U^{n}$ and $g(x)=f(p(x))-f(-p(x))$ for $x \in V^{n} \backslash U^{n}$. Then, it is easy to show that $g(x)=-g(-x)$ for any $x \in \mathbb{Z}^{n} \cap \operatorname{Bd}\left(V^{n}\right)$. We now have the following lemma.

Lemma 4.3 Let $f: \mathbb{Z}^{n} \rightarrow \mathbb{R}^{n}$ be a locally gross direction preserving function satisfying Assumption 4.1. Then $g$ is locally gross direction preserving on $\mathbb{Z}^{n} \cap V^{n}$.

Proof. Clearly, $g$ is locally gross direction preserving for any cell-connected points in $U^{n}$. It remains to consider the following two cases.

First, let $x, y \in \mathbb{Z}^{n}$ be two cell-connected points on the boundary of $V^{n}$. Then $p(x)$ and $p(y)$ are cell-connected and lie on a same proper face of $U^{n}$, so that

$$
\begin{aligned}
g(x) \cdot g(y)= & (f(p(x))-f(-p(x))) \cdot(f(p(y))-f(-p(y))) \\
= & f(p(x)) \cdot f(p(y))-f(p(x)) \cdot f(-p(y))-f(-p(x)) \cdot f(p(y)) \\
& +f(-p(x)) \cdot f(-p(y)) \geq 0,
\end{aligned}
$$

where the inequality follows from both the locally gross direction preservation of $f$ and the boundary condition.

Second, let $x, y \in \mathbb{Z}^{n}$ be two cell-connected points with $x$ on the boundary of $U^{n}$ and $y$ on the boundary of $V^{n}$. Then $x$ and $p(y)$ are two cell-connected points and lie on a same proper face of $U^{n}$, so that

$$
\begin{aligned}
g(x) \cdot g(y) & =f(x) \cdot(f(p(y))-f(-p(y))) \\
& =f(x) \cdot f(p(y))-f(x) \cdot f(-p(y)) \geq 0,
\end{aligned}
$$

where the inequality again follows from both the locally gross direction preservation of $f$ and the boundary condition.

We are now ready to prove Theorem 4.2 by using a symmetric integral triangulation of $\mathbb{R}^{n}$ in the sense that if $\sigma$ is a simplex of the triangulation, then $-\sigma$ is also a simplex of the triangulation. For instance, the $K^{\prime}$-triangulation of $\mathbb{R}^{n}$ introduced in Todd [28] is a symmetric integral triangulation.

Proof of Theorem 4.2. To prove the theorem, let the set $V^{n}$ and the function $g$ be defined as above. Take the origin $0^{n}$ of $\mathbb{R}^{n}$ as the starting point $v$ of the algorithm as described in Section 2 and take a symmetric integral triangulation $\mathcal{T}$ of $V^{n}$.

Starting with the origin, the algorithm generates a sequence of adjacent almost $s$ complete simplices with $s$-complete common facets in $A(s) \cap V^{n}$ for varying sign vectors $s$ with the following modification. If $\tau$ is an $s$-complete facet lying in $A(s)$ on the boundary of $V^{n}$, then the antipodal facet $-\tau$ is a $(-s)$-complete facet in $A(-s)$ on the boundary of $V^{n}$, 
since $g(x)=-g(-x)$ for any $x \in \mathbb{Z}^{n} \cap \operatorname{Bd}\left(V^{n}\right)$. The algorithm continues in $A(-s) \cap V^{n}$ with the unique almost $(-s)$-complete simplex in this set containing $-\tau$ as facet. The algorithm therefore always stays in $V^{n}$ and due to the lexicographic pivoting rule will never visit any simplex in $V^{n}$ more than once. Since the number of simplices in $V^{n}$ is finite, within a finite number of steps the algorithm terminates with a complete simplex $\sigma^{*}$ in $V^{n}$. Since $g$ is locally gross direction preserving and thus also simplicially local gross direction preserving on $V^{n}$, by the Lemmas 2.3 and 4.3 it follows that $\sigma^{*}$ has a vertex $z$ being a discrete zero point of $g$. It remains to prove that $p(z) \in U^{n}$ is a discrete zero point of $f$. If $z$ is not on the boundary of $V^{n}$, then $z=p(z)$ is an integral vector in $U^{n}$ and $f(z)=g(z)$. Since $g(z)=0^{n}$, this implies that $z$ is a discrete zero point of $f$. Suppose $z$ is on the boundary of $V^{n}$. Since $g(z)=0^{n}$, this implies

$$
\begin{aligned}
0 & =f(p(z)) \cdot g(z)=f(p(z)) \cdot(f(p(z))-f(-p(z))) \\
& =f(p(z)) \cdot f(p(z))-f(p(z)) \cdot f(-p(z)),
\end{aligned}
$$

and therefore

$$
0 \leq f(p(z)) \cdot f(p(z))=f(p(z)) \cdot f(-p(z)) \leq 0
$$

where the last inequality follows from the boundary condition on $f$. Hence, $f(p(z))$. $f(p(z))=0$ and therefore $p(z)$ is a discrete zero point of $f$ in $U^{n}$.

\section{Integral Solution of Complementarity Problems}

The complementarity problem is to find a point $x^{*} \in \mathbb{R}^{n}$ such that

$$
x^{*} \geq 0^{n}, f\left(x^{*}\right) \geq 0^{n}, \text { and } x^{*} \cdot f\left(x^{*}\right)=0,
$$

where $f$ is a given function from $\mathbb{R}^{n}$ into itself. Specifically, if $f$ is affine, the problem is called the linear complementarity problem. In general, for an arbitrary function $f$, the problem is called the nonlinear complementarity problem. If the solution of the complementarity problem is required to be integral or if the function $f$ is defined only on the integer lattice $Z^{n}$ of $\mathbb{R}^{n}$, then we call the problem the discrete complementarity problem, denoted by $\operatorname{DCP}(f)$. There is by now a voluminous literature on the complementarity problem, see Lemke [21], Cottle [2], Eaves [5], Karamardian [12], Moré [23], [24], Kojima [13], van der Laan and Talman [18] among others. For comprehensive surveys on the subject, see for example Kojima et al. [14], Cottle et al. [3], Facchinei and Pang [8].

In this section we give a sufficient condition under which $\operatorname{DCP}(f)$ has a solution. For any $x, y \in \mathbb{R}^{n}$, let $I(x)=\left\{i \mid x_{i}>0\right\}$ and let $I(x, y)=I(x) \cup I(y)$. 
Definition 5.1 A function $f: \mathbb{Z}^{n} \rightarrow \mathbb{R}^{n}$ is simplicially local gross direction preserving on $\mathbb{Z}_{+}^{n}$ with respect to some integral triangulation $\mathcal{T}$ of $\mathbb{R}^{n}$, if for any two vertices $x$ and $y$ of any simplex of $\mathcal{T}$ in $\mathbb{R}_{+}^{n}$ it holds that

$$
f_{i}(x) f_{i}(y) \geq 0, \text { for } i \notin I(x, y)
$$

and

$$
\sum_{h \in I(x, y)} f_{h}(x) f_{h}(y) \geq 0
$$

Theorem 5.2 Let $f: \mathbb{Z}^{n} \rightarrow \mathbb{R}^{n}$ be a simplicially local gross direction preserving function on $\mathbb{Z}_{+}^{n}$. When there exists a vector $M \in \mathbb{Z}_{++}^{n}$ such that for any $x \in \mathbb{Z}_{+}^{n}, x_{i}=M_{i}$ implies $f_{i}(x) \geq 0$, then $D C P(f)$ has a solution.

To prove the theorem we adapt the algorithm described in Section 2 to the current problem by making several modifications. First, the origin $0^{n}$ of the set $\mathbb{R}^{n}$ is chosen to be the starting point $v$ of the algorithm. Since $0^{n}$ lies on the boundary of $\mathbb{R}_{+}^{n}$, the sets $A(s)$ and $s$-completeness are only defined for nonnegative sign vectors $s$. Notice that for such sign vector $s$ it holds that $A(s)=\left\{x \in \mathbb{R}_{+}^{n} \mid x_{i}=0\right.$ whenever $\left.s_{i}=0\right\}$.

Next, we adapt the concepts of an almost $s$-complete simplex and an $s$-complete facet. For some nonnegative (nonzero) sign vector $s$ with $t$ positive components, denote $\left\{h_{1}, \ldots, h_{n-t}\right\}=\left\{h \mid s_{h}=0\right\}$ and let $\sigma=<x^{1}, \cdots, x^{t+1}>$ be a $t$-simplex of the triangulation in $A(s)$. Then $\sigma$ is almost s-complete if there is an $(n+2) \times(n+1)$ matrix $W$ being a solution to system

$$
\left[\begin{array}{ccccccc}
1 & \cdots & 1 & 0 & \cdots & 0 & 1 \\
f\left(x^{1}\right) & \cdots & f\left(x^{t+1}\right) & -e\left(h_{1}\right) & \cdots & -e\left(h_{n-t}\right) & s
\end{array}\right] W=I
$$

and having rows $w^{1}, \cdots, w^{n+2}$ such that $w^{h} \succeq 0$ for $1 \leq h \leq t+1, w^{n+2} \succeq-w^{i}$ for $t+1<i \leq n+1$ and $w^{n+2} \succeq 0$. If $w_{1}^{n+2}=0$, then we say that the simplex $\sigma$ is complete. A facet $\tau$ of $\sigma$, without loss of generality given by $\tau=\left\langle x^{1}, \cdots, x^{t}\right\rangle$, is $s$-complete if there is an $(n+1) \times(n+1)$ matrix $W$ being a solution to system

$$
\left[\begin{array}{ccccccc}
1 & \cdots & 1 & 0 & \cdots & 0 & 1 \\
f\left(x^{1}\right) & \cdots & f\left(x^{t}\right) & -e\left(h_{1}\right) & \cdots & -e\left(h_{n-t}\right) & s
\end{array}\right] W=I
$$

and having rows $w^{1}, \cdots, w^{n+1}$ such that $w^{h} \succeq 0$ for $1 \leq h \leq t, w^{n+1} \succeq-w^{i}$ for $t+1 \leq i \leq n$ and $w^{n+1} \succeq 0$. If $w_{1}^{n+1}=0$, then we say that $\tau$ is complete.

With respect to the starting point $0^{n}$, let $\alpha=\min _{h} f_{h}\left(0^{n}\right)$. If $\alpha \geq 0$ then $0^{n}$ solves $\operatorname{DCP}(f)$ and the algorithm terminates. Suppose the origin does not solve $\operatorname{DCP}(f)$, i.e. $\alpha<0$, then let $s^{0}$ be the sign vector with $s_{k}^{0}=1$, where $k$ is the smallest index $h$ such 
that $f_{h}\left(0^{n}\right)=\alpha$, and $s_{j}^{0}=0$ for $j \neq k$. Similarly as in Section 2, it can be shown that the simplex $<0^{n}>$ is an $s^{0}$-complete facet of the unique 1-dimensional simplex $\sigma^{0}$ in $A\left(s^{0}\right)$ having $<0^{n}>$ as one of its facets. Furthermore $\sigma^{0}$ is almost $s^{0}$-complete.

We now apply the algorithm as described in Section 2. Let $C^{n}=\left\{x \in \mathbb{R}_{+}^{n} \mid\right.$ $x \leq M\}$ and let $\mathcal{T}$ be the integral triangulation of $\mathbb{R}^{n}$ with respect to the function $f$. This triangulation subdivides $\mathbb{R}_{+}^{n}$ and the set $A(s)$ for every nonnegative sign vector $s$ into $t$-dimensional integral simplices. Starting with $\sigma^{0}$, the algorithm generates a unique sequence of adjacent almost $s$-complete simplices in $A(s)$ with $s$-complete common facets, for varying nonnegative sign vectors $s$. The algorithm stops when an $s$-complete facet on the upper boundary of $C^{n}$ is generated or when a complete simplex in $A(s)$ is found. The next lemma says that in the first case the facet must be complete.

Lemma 5.3 For some nonnegative sign vector s, let $\tau$ be an s-complete facet of an almost s-complete simplex in $A(s) \cap C^{n}$, lying on the upper boundary of $C^{n}$. Then $\tau$ is a complete facet.

Proof. Let $\tau=<x^{1}, \cdots, x^{t}>$, where $t$ is the number of positive components of $s$. From system (5.4) it follows that there exist $\lambda_{1} \geq 0, \cdots, \lambda_{t} \geq 0$ with sum equal to one, $\beta \geq 0$, and $\mu_{i} \geq-\beta$ for $s_{i}=0$, such that $\bar{f}_{i}(z)=-\beta$ when $s_{i}=1$ and $\bar{f}_{i}(z)=\mu_{i}$ when $s_{i}=0$, where $z=\sum_{i=1}^{t} \lambda_{i} x^{i}$ and $\bar{f}$ is the piecewise linear extension of $f$ on $\mathbb{R}^{n}$. Since $\tau$ lies on the upper boundary of $C^{n}$, there exists an index $h$ such that $x_{h}^{j}=M_{h}$ for all $j$. But then we must have $s_{h}=1$ and therefore $\bar{f}_{h}(z)=-\beta \leq 0$. On the other hand, by assumption on $f$, it holds that $f_{h}\left(x^{j}\right) \geq 0$ for all $j$. Hence, we obtain $\bar{f}_{h}(z)=\sum_{j=1}^{t} \lambda_{j} f_{h}\left(x^{j}\right) \geq 0$. Therefore $w_{1}^{n+1}=\beta=0$, i.e. $\tau$ is complete.

The lemma implies that if the origin does not solve the problem, the algorithm only stops with a complete simplex or facet. The next lemma says that at least one of the vertices of any complete simplex or facet solves the discrete complementarity problem.

Lemma 5.4 For some nonnegative sign vector s, let $\sigma$ be a complete simplex or facet in $A(s)$. Then $\sigma$ contains a vertex being a solution to $\operatorname{DCP}(f)$.

Proof. Let $\sigma=<x^{1}, \cdots, x^{h+1}>$ be a complete $h$-simplex in $A(s)$, where $h=t$ or $t+1$ and $t$ is the number of positive components of $s$. From (5.4) in case $h=t$ or (5.3) in case $h=t+1$ it follows that there exist $\lambda_{1} \geq 0, \cdots, \lambda_{h+1} \geq 0$ with sum equal to one and $\mu_{i} \geq 0$ for $s_{i}=0$ such that $\bar{f}_{i}(z)=0$ if $s_{i}=1$, and $\bar{f}_{i}(z)=\mu_{i}$ if $s_{i}=0$, where $z=\sum_{i=1}^{h+1} \lambda_{i} x^{i}$. Since $z \in A(s)$, we also have $z_{i}=0$ if $s_{i}=0$ and $z_{i} \geq 0$ if $s_{i}=1$. So, $\bar{f}_{i}(z) \geq 0$ if $z_{i}=0$ and $\bar{f}_{i}(z)=0$ if $z_{i}>0$, i.e., $z$ solves the nonlinear complementarity problem with respect to $\bar{f}$. Now, let $\rho=<y^{1}, \ldots, y^{k}>$ be the unique $(k-1)$-face of $\sigma$ containing $z$ in its 
relative interior. Hence, there exist unique positive numbers $\lambda_{1}, \ldots, \lambda_{k}$ summing up to 1 such that $z=\sum_{j=1}^{k} \lambda_{j} y^{j}$ and $\bar{f}(z)=\sum_{j=1}^{k} \lambda_{j} f\left(y^{j}\right)$. Take any $j^{*}$ between 1 and $k$. Suppose first that $z_{i}=0$ and $\bar{f}_{i}(z)>0$ for some $i$. Clearly, $y_{i}^{j}=0$ for all $j=1, \ldots, k$. Since $\bar{f}_{i}(z)=\sum_{j=1}^{k} \lambda_{j} f_{i}\left(y^{j}\right)$ there exists $h$ such that $f_{i}\left(y^{h}\right)>0$. Since $y^{h}$ and $y^{j^{*}}$ are simplicially connected and $y_{i}^{h}=y_{i}^{j^{*}}=0$, we have that $f_{i}\left(y^{h}\right) f_{i}\left(y^{j^{*}}\right) \geq 0$, and therefore $y_{i}^{j^{*}}=0$ and $f_{i}\left(y^{j^{*}}\right) \geq 0$. Suppose next that $z_{i}=0$ and $\bar{f}_{i}(z)=0$ for some $i$. Again, $y_{i}^{j}=0$ for all $j=1, \ldots, k$. Since $\bar{f}_{i}(z)=\sum_{j=1}^{k} \lambda_{j} f_{i}\left(y^{j}\right)$ and $\bar{f}_{i}(z)=0$, we obtain $\sum_{j=1}^{k} \lambda_{j} f_{i}\left(y^{j}\right)=0$ and therefore $\sum_{j=1}^{k} \lambda_{j} f_{i}\left(y^{j}\right) f_{i}\left(y^{j^{*}}\right)=0$. Since for all $j$ it holds that $y^{j}$ and $y^{j^{*}}$ are simplicially connected and $y_{i}^{j}=y_{i}^{j^{*}}=0$, we have $f_{i}\left(y^{j}\right) f_{i}\left(y^{j^{*}}\right) \geq 0$, and so each term in the summation must be zero. In particular, it holds that $\lambda_{j^{*}} f_{i}^{2}\left(y^{j^{*}}\right)=0$. Since $\lambda_{j^{*}}>0$, this implies $f_{i}\left(y^{j^{*}}\right)=0$.

Thus far we have shown that whenever $z_{i}=0$ both $f_{i}\left(y^{j^{*}}\right) \geq 0$ and $y_{i}^{j^{*}}=0$ must hold. It remains to show that for $z_{i}>0$ it holds that $y_{i}^{j^{*}} \geq 0$ and $f_{i}\left(y^{j^{*}}\right)=0$, and hence that $y^{j^{*}}$ is a solution to $\operatorname{DCP}(f)$. Clearly, $y_{i}^{j^{*}} \geq 0$ if $z_{i}>0$. Moreover, $\bar{f}_{i}(z)=\sum_{j=1}^{k} \lambda_{j} f_{i}\left(y^{j}\right)=0$ whenever $z_{i}>0$. Therefore,

$$
\sum_{h \in I(z)} \sum_{j=1}^{k} \lambda_{j} f_{h}\left(y^{j}\right) f_{h}\left(y^{j^{*}}\right)=0
$$

and so

$$
\sum_{j=1}^{k}\left(\lambda_{j} \sum_{h \in I(z)} f_{h}\left(y^{j}\right) f_{h}\left(y^{j^{*}}\right)\right)=0 .
$$

Since $I(z)$ contains the set $I\left(y^{j}, y^{j^{*}}\right)$ and $y^{j}$ and $y^{j^{*}}$ are simplicially connected for all $j$, by hypothesis we have that each of the $k$ terms between brackets is nonnegative and therefore must be zero. Hence,

$$
\lambda_{j^{*}} \sum_{h \in I(z)} f_{h}^{2}\left(y^{j^{*}}\right)=0
$$

Since $\lambda_{j^{*}}>0$, we obtain $f_{h}\left(y^{j^{*}}\right)=0$ for all $h \in I(z)$. Therefore $f_{i}\left(y^{j^{*}}\right)=0$ and $y_{i}^{j^{*}} \geq 0$ if $z_{i}>0$, which completes the proof.

Theorem 5.2 now follows from the two lemmas stated above by a combinatorial argument.

Proof of Theorem 5.2. For varying nonnegative sign vectors $s$, the algorithm generates a sequence of almost $s$-complete simplices in $A(s)$ with $s$-complete common facets. The algorithm cannot leave $C^{n}$, because according to Lemma 5.3 it stops with a complete facet when it generates an $s$-complete facet on the upper bound of $C^{n}$. Due to the lexicographic 
pivoting rule, the algorithm will never visit any simplex more than once. Since the number of simplices in $C^{n}$ is finite, the algorithm therefore must terminate within a finite number of steps with a complete simplex or facet in $C^{n}$. According to Lemma 5.4 one of its vertices is a solution to $\operatorname{DCP}(f)$.

\section{References}

[1] Allgower, E.L., Georg, K., 1990. Numerical Continuation Methods: An Introduction, Springer, Berlin.

[2] Cottle, R.W., 1966. Nonlinear programs with positively bounded Jacobians. SIAM Journal on Applied Mathematics 14, 147-158.

[3] Cottle, R.W., Pang, J.-S., Stone, R.E., 1992. The Linear Complementarity Problem, Academic Press, New York.

[4] Danilov, V., Koshevoy, G., 2004. Existence theorem of zero point in a discrete case, Moscow, draft.

[5] Eaves, B.C., 1971. On the basic theorem of complementarity. Mathematical Programming 1, 68-75.

[6] Eaves, B.C., 1972. Homotopies for computation of fixed points, Mathematical Programming 3, 1-22.

[7] Eaves, B.C., Saigal, R., 1972. Homotopies for computation of fixed points on unbounded regions, Mathematical Programming 3, 225-237.

[8] Facchinei, F., Pang, J.-S., 2003. Finite-Dimensional Variational Inequalities and Complementarity Problems, Vol. I, Vol. II, Springer, New York.

[9] Freund, R.M., Todd, M.J., 1981. A constructive proof of Tucker's combinatorial lemma, Journal of Combinatorial Theory 30, 321-325.

[10] Iimura, T., 2003. A discrete fixed point theorem and its applications, Journal of Mathematical Economics 39, 725-742.

[11] Iimura, T., Murota, K., Tamura, A., 2004. Discrete fixed point theorem reconsidered, METR 2004-09, University of Tokyo, Tokyo, forthcoming in Journal of Mathematical Economics. 
[12] Karamardian, S., 1972. The complementarity problem. Mathematical Programming 2, 107-129.

[13] Kojima, M., 1975. A unification of the existence theorems of the nonlinear complementarity problem. Mathematical Programming 9, 257-277.

[14] Kojima, M., Megiddo, N., Noma, T., Yoshise, A., 1991. A Unified Approach to Interior Point Algorithms for Linear Complementarity Problems, Springer-Verlag, Berlin.

[15] Laan van der, G., 1984. On the existence and approximation of zeros, Mathematical Programming 28, 1-14.

[16] Laan van der, G., Talman, A.J.J., 1979. A restart algorithm for computing fixed points without an extra dimension, Mathematical Programming 17, 74-84.

[17] Laan van der, G., Talman, A.J.J., 1981. A class of simplicial restart fixed point algorithms without an extra dimension, Mathematical Programming 20, 33-48.

[18] Laan van der, G., Talman, A.J.J., 1987. Simplicial approximation of solutions to the nonlinear complementarity problem. Mathematical Programming 38, 1-15.

[19] Laan van der, G., Talman, A.J.J., Yang, Z., 2004. Solving discrete zero point problems, TI discussion paper 04-112/1, forthcoming in Mathematical Programming.

[20] Laan van der, G., Talman, A.J.J., Yang, Z., 2005. Computing integral solutions of complementarity problems, TI discussion paper 2005-006/1, Amsterdam.

[21] Lemke, C.E., 1965. Bimatrix equilibrium points and mathematical programming. Management Science 11, 681-689.

[22] Merrill, O.H., 1972. Applications and Extensions of an Algorithm that Computes Fixed Points of Certain Upper Semi-Continuous Point-to-Set Mappings, PhD Thesis, University of Michigan, Ann Arbor.

[23] Moré, J.J., 1974a. Coercivity conditions in nonlinear complementarity problem. SIAM Review 17, 1-16.

[24] Moré, J.J., 1974b. Classes of functions and feasibility conditions in nonlinear complementarity problem. Mathematical Programming 6, 327-338.

[25] Reiser, P.M., 1981. A modified integer labeling for complementarity algorithms, Mathematics of Operations Research 6, 129-139. 
[26] Scarf, H., 1967. The approximation of fixed points of a continuous mapping, SIAM Journal on Applied Mathematics 15, 1328-1343.

[27] Todd, M.J., 1976. Computation of Fixed Points and Applications, Springer-Verlag, Berlin.

[28] Todd, M.J., 1978. Improving the convergence of fixed point algorithms, Mathematical Programming Study 7, 151-179.

[29] Todd, M.J., 1980. Global and local convergence and monotonicity results for a recent variable-dimension simplicial algorithm, in Numerical Solution of Highly Nonlinear Problems, edited by W. Forster, North-Holland, Amsterdam.

[30] Todd, M.J., Wright, A.H., 1980. A variable dimension simplicial algorithm for antipodal fixed point theorems, Numerical Functional Analysis and Optimization 2, 155-186.

[31] Tucker, A.W., 1945. Some topological properties of disk and sphere, Proceedings of the First Canadian Mathematical Congress, Montreal, 285-309.

[32] Yang, Z., 1999. Computing Equilibria and Fixed Points, Kluwer, Boston.

[33] Yang, Z., 2004a. Discrete nonlinear complementarity problems, FBA Working Paper No. 205, Yokohama National University, Yokohama.

[34] Yang, Z., 2004b. Discrete fixed point analysis and its applications, FBA Working Paper No. 210, Yokohama National University, Yokohama. 


\section{Tinbergen Institute}

The Tinbergen Institute is the institute for economic research of the Erasmus Universiteit Rotterdam, Universiteit van Amsterdam, and Vrije Universiteit Amsterdam.

Tinbergen Institute Amsterdam

Roetersstraat 31

1018 WB Amsterdam

The Netherlands

Tel.: $\quad+31(0) 205513500$

Fax: $\quad+31(0) 205513555$

Tinbergen Institute Rotterdam

Burg. Oudlaan 50

3062 PA Rotterdam

The Netherlands

Tel.: $\quad+31(0) 104088900$

Fax: $\quad+31(0) 104089031$

Please send questions and/or remarks of nonscientific nature to driessen@tinbergen.nl.

Most TI discussion papers can be downloaded at http://www.tinbergen.nl. 